\title{
A ISO 9001:2000 Certification Model in SMEs
}

\author{
Raimundo Sales Neto e Azevedo \\ Arnaldo Dias Belchior \\ Universidade de Fortaleza, Mestrado em Informática Aplicada, \\ Fortaleza-Ceará, Brazil, 60811-341 \\ sales.mia@unifor.br, belchior@unifor.br \\ and \\ Marum Simão Filho \\ Flávio Lenz César \\ SoftExport \\ Fortaleza-Ceará, Brazil, 60811-341 \\ marum@softexport.com.br, lenz@softexport.com.br
}

\begin{abstract}
This work describes a model for certification ISO 9001:2000 process of a software company. The Quality Management System (QMS) implementation in the company guided the fundamental processes of the software development, the organizational processes and the support processes, structuring all the practices in the company and extending its vision in relation to their own products, processes, collaborators and clients. The certification process was conducted through a set of structured activities based upon ISO/IEC 12207, in contrast to the logical sequence of implementation items in ISO 9001. As a result, this work provided greater agility in the implementation process of the QMS in the software company.
\end{abstract}

Keywords: software process, ISO certification, ISO 9001, ISO/IEC 12207, ISO/IEC 15271 


\section{INTRODUCTION}

ISO 9000 Certification [1] is recognized throughout the world in various production sectors. The conquest of ISO certification takes the organization to a level of international standard in the quality of its processes. However, in the scope of one determined sector, it is not possible to differentiate the level of maturity of a company in relation to another one, in a set of companies who have received ISO 9000 certification, apart from by the scope of the certification, the quality of the certifier and the time for which the certification is being maintained [5, 6].

ISO 9000 is an international standard of quality that applies to the management of the quality of the process used to generate products that attend to the expectations of its customers. These standards were created under the premise that if the development and the management of the system are of good quality, then the product or the resultant service also will be of good quality. A Quality Management System (QMS) in compliance with ISO 9000 will assure that its process of development has a disciplined, repeatable level of control, guaranteeing the quality of its products.

In Brazil, according to the data obtained by Paduan [3], at the end of the 2000 there existed 5,400 companies involved in the production and commercialization of software, with a total of 158,000 employees. By performing a simple calculation, we can observe that on average, there are about 29 employees in each company. This evidences suggests that the majority of national companies in the area of software development are of small or medium size. They consider a small company to be up to 49 employees and a medium size up to 99 employees.

Table 1: MCT/Quality and Productivity in the Sector of Brazilian Software - 2001

\begin{tabular}{|l|l|}
\hline \multicolumn{1}{|c|}{ Scale } & \multicolumn{1}{c|}{ Zone } \\
\hline Micro & $1-9$ employees \\
\hline Small & $10-49$ employees \\
\hline Medium & $50-99$ employees \\
\hline Large & $100-+$ employees \\
\hline
\end{tabular}

Associated with this number, we have organizations whose software development is not a final activity. Therefore, the generated software does not have the characteristics of a commercial product and is for internal use.

In both the mentioned sectors, Small and Medium Enterprises (SME) and Internal Development Organizations (IDO), do not have justifications for not pursuing the development of quality products, with detailed documented procedures, registers, supervision, historical generated data and conducted by an institutional policy, that is followed by all, and that evolves with the maturity of the processes. To introduce a QMS that associates operational aspects of quality assurance with its management and strategic aspects is indispensable.

SME have the following characteristics:

a Management accumulates roles in the development processes and inevitably have to assume administrative roles;

- Few financial resources;

․ High turnover of collaborators;

a Accumulation of roles and responsibilities;

口 Source of experts and expertise;

a Increasing customer requirements for quality products that are delivered on time and within the specified budget.

a The existence of products developed internally with commercial potential.

Often, this reduced organizational structure is shocked by maturity models, which generally indicate a heavy structure, densely populated by innumerable functions and responsibilities [8]. In IDO in general, there is no specific interest for a certification, as the interest lies in the effects of the implantation of a Quality Management System (QMS) in the reduction of costs and an increase in the quality of the software.

The generic approach of norms ISO 9000 and ISO 9001[2] makes the proposed Quality Management System applicable to any organization with processes that receive components whose entrance were the result of intermediate or final products. This approach however becomes excessively generic to be applied in software SME, where a large amount of the components of the organization, people, processes and activities are directly liked to the software production routine. When the organization has documents relating to its products and customers, the 
development processes and methodologies already exist in some form and are being applied. The people who participate in the company, the directors, the project managers, the system analysts, the designers, the developers, the test analysts and the support work together and perform the development routines on a daily basis. The greatest difficulty for this group is the agreement the organizational vision. The administrative and financial routines must be attended to and they are not simple routines.

The approach of ISO/IEC 12207 Norm [4] and its description of software lifecycle, brings together the organization environment and its daily software development activities with the system, processes, activities and tasks concepts in a clear way for all in organization.

The proposal of this work is the adoption of ISO 9001:2000 (ISO 9001) norm structure for the introduction of a QMS to generate products and for creation or organization of quality culture in SME using the described structure in ISO/IEC 12207 Norm.

The perception of a standard is a indicative that had an agreement. The analyzed element of some form incased in a known and dominated behavior. The ISO/IEC 12207 standard, known and with vocabulary applied in software development routines, is integrated with the ISO 9001 standard, which is generic, long range and distant from dayby-day of the organization in direct application. According to Campos [9], the term 'standard' mentions everything that unifies and simplifies for people benefits, adopted for consensus and capable to modify, including procedures, concepts and also measurement methods.

\section{THE ISO 9000:2000 AND ISO 9001:2000 NORMS}

The ISO 9000 [1] and ISO 9001[2] Norms were elaborated to guide organizations in the efficient implementation of a QMS. Given the implementation of this system, whose focus is on quality assurance, the company will be prepared to deal with the occurrence of problems with a greater agility and effectiveness. The quality will be reflected in its processes and products in a continuously improving action.

The ISO 9000 Norm defines the fundamentals and vocabulary to define the QMS. For management and those involved in the control of an organization, the high level management has to have a systemic vision of all processes and activities, identifying the points of influence and the reach of the entire organizational structure, integrally involving customers and suppliers in the institutional processes. In support to these necessities the ISO 9000 Norm identifies eight principles for quality management:

- Customer focus;

- Leadership;

- People involvement;

口 Process approach;

- Systemic management approach;

- Continuous improvement;

- Information-based decisions;

- Mutual benefits in the relationships with suppliers.

The integrated application of these eight principles requires various stages to develop and implement a QMS:

- Determination of the necessities and expectations of the customers and other interested people;

口 Establishment of quality policies and objectives of the organization;

a Determination of the processes and responsibilities necessary to achieve the quality objectives;

口 Determination of the supply of resources necessary to achieve the quality objectives;

a Establishment of methods to determine the effectiveness and efficiency of each process;

- Application of these measures to determine the effectiveness and efficiency of each process;

- Determination of the methods to prevent not-conformities and eliminating their causes;

\ Establishment and application of improvement processes for the QMS;

In the following transcribed section of the ISO 9000 Norm we can identify another approach of the processes: "for the organization to function in an efficient form, they have to identify and manage interrelated and interactive processes. Frequently, the output of a process will result directly in the entrance of the following process. The 
systematic identification and the process management used in the organization and, particularly, the interactions between such processes are known as 'processes approach'”. This approach is represented in figure 1.

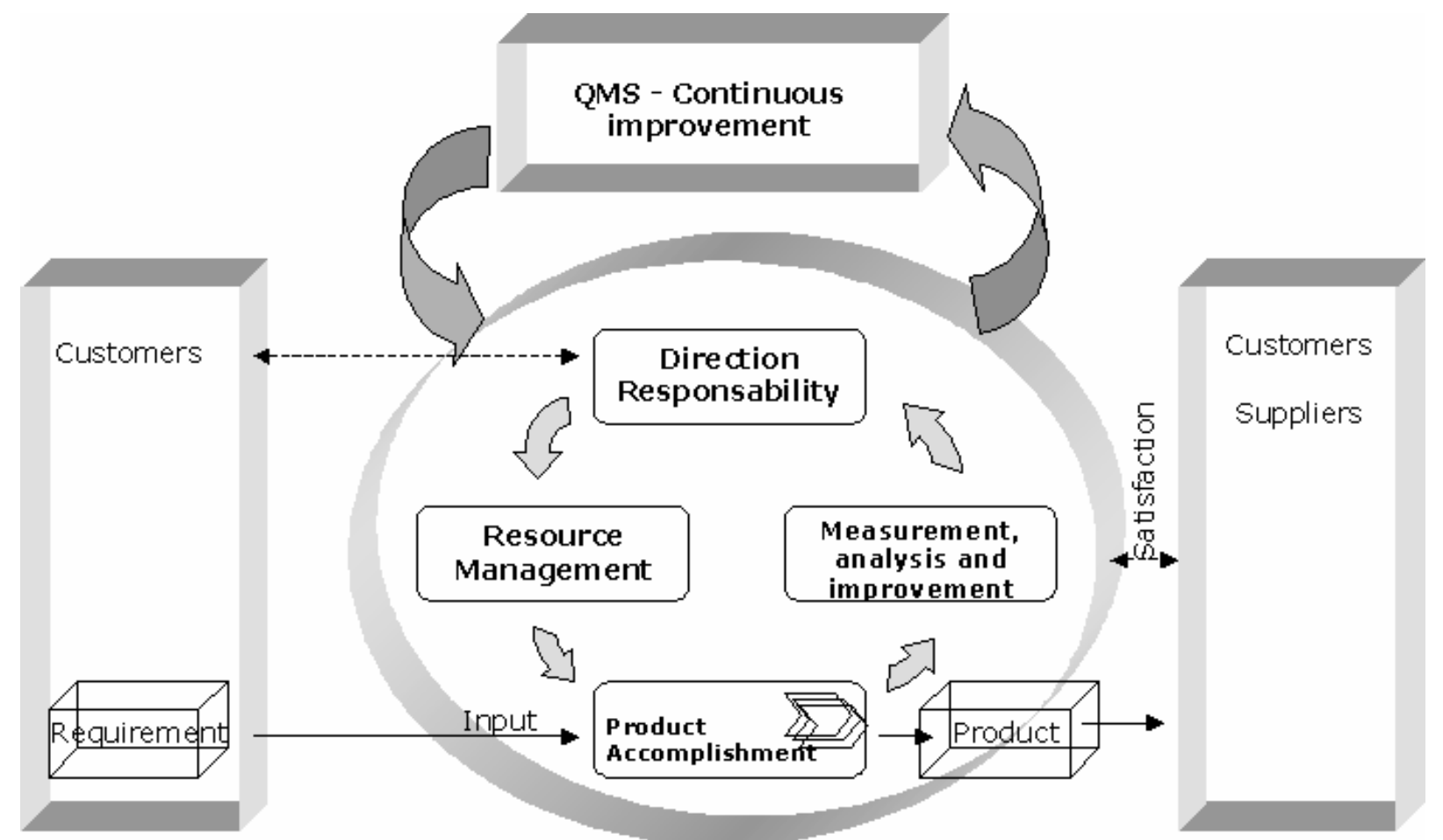

Figure 1: QMS-based process Model

The QMS of a company possess a set of directives that allows its customers to evaluate the capacity of the organization to supply products and services, which attend to the specified requirements in a consistent form and still provide a continuous improvement structure. An organization that intends to certify its QMS based on the ISO 9001 Norm will need to consider the following questions:

- To know and demonstrate its capacity in attending to customer requirements;

To plan and document all the activities that affect quality;

- To identify people with the competencies necessary to accomplish tasks;

- To identify and make available the human and material resources necessary to maintain the QMS;

- To register the executed activities;

- To prevent not-conformities and when they occur, they must be registered and handled.

- To identify the critical processes to obtain customer satisfaction;

- To maintain a continuous program of evaluation of the performance of the system.

The organization must apply its quality policies to prove that the high level management is committed to the QMS. The quality policies must be adequate for the purpose of the organization, to be genuine and to reflect the values of the company to the customers, employees and interested parties.

The ISO 9001 Norm recommends that for an efficient management, the best form to attend to requirements is the creation of registered procedures. The procedures are specified in a form to develop an activity. The Norm demands only the elaboration of six procedures:

- Document control;

- Record Control;

- Internal audit;

- Not-conformity control;

- Corrective action;

口 Preventive action. 
The existence of procedures, instructions and documents describing work performed, formalizes all the activities that affect quality. This demands the participation of all individuals in the organization. Therefore, once everybody is participating directly in the implementation of the QMS, the awareness of quality increases, as they are the responsible for the activities within the organization.

The customer influences the beginning, the actual process and the end product. The control of a software development procedure and of the management of the QMS leads to the control of the production process, which can guarantee the quality of the final product. The QMS is the support for the conformity of this norm and must be structured to control and publish the set of procedures used by the organization, to facilitate and promote change management and to facilitate the activities of monitoring and audit of the QMS.

For an organization to be certified according to ISO 9001, it must show that it understands the customer requirements, to plan and register the activities that affect quality, to evaluate people and to identify and make available resources to maintain the QMS. It must register its activities, prevent not-conformities, register their occurrences and handle their cause, besides keeping a continuous program of evaluation of the QMS, for the continuous improvement of all processes.

\section{THE ISO/IEC 12207:1995 AND ISO/IEC 15271:1998 NORMS}

The architecture of the ISO/IEC 12207 [4] Norm uses a systemic approach with the concept of processes (set of interrelated activities that transform inputs into outputs), activities (includes the use of resources) and tasks (expressed in the form of a requirement, self-explanatory, recommendation or allowed action). It describes the software life cycle in three macro-processes:

a Fundamental processes: These group the processes that directly integrate with the software production. These being Acquisition, Supply, Development, Operation and Maintenance.

a Support processes: The support processes assist other processes in the search of success and quality of the project and are formed by Documentation, Configuration Management, Quality Guarantee, Verification, Validation, Joint Revision, Auditing and Problem Resolution.

- Organizational processes: The organizational processes typically involve institutional practices and policies and are composed of Management, Infrastructure, Training and Improvement.

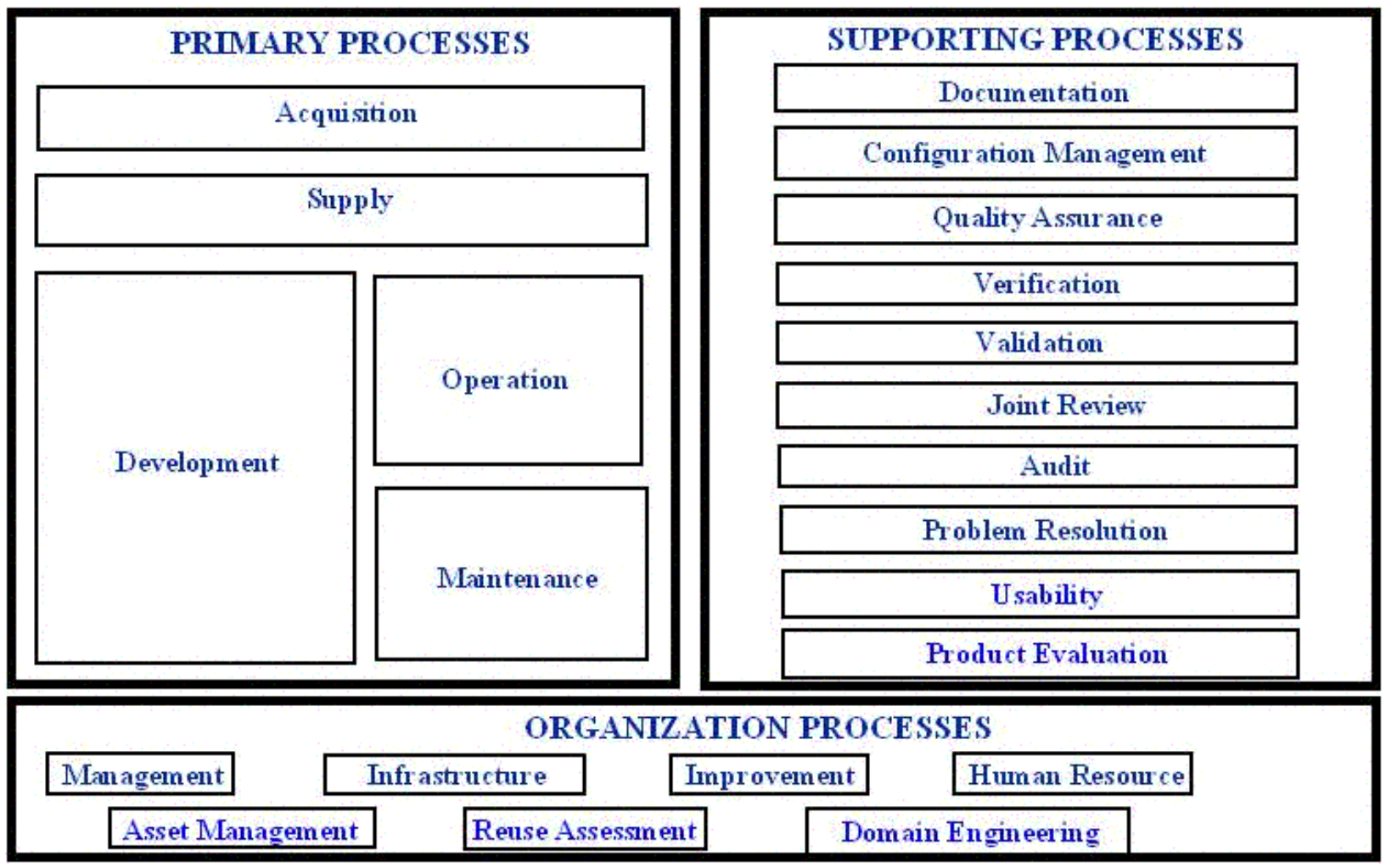

Figure 2: Software Life Cycle Processes ISO/IEC 12207 / Amd 1:2002[10]

Each macro-process is composed of processes that are composites of a set of activities that are in turn composed of a set of tasks. The application of the processes of this Norm constitute a wide and generic set. Each 
organization can select the subgroup appropriate for its activities, and this can be adapted for specific projects or applications.

The structure incorporated by the Norm is also appropriate when the software is an independent entity, an embedded entity or an entity integrated within a system (jointly integrated consisting of one or more processes, the hardware, software, resources and people, capable of satisfying a necessity or a defined objective).

In the ISO 12207 Norm, there exists the annex A. Adaptation Process (normative) and B. Orientation for adaptation (informative). Foreseeing the variations in organizational policies and procedures, methods and strategies of acquisition, the size and complexity of the projects and requirements and system development methods, these annexes suggest that all the parties involved in the project would need to be involved in this adaptation to the reality of the organization, following the defined rules and relationships.

According to the ISO/IEC 15271 Norm [7] "the ISO/IEC 12207 Norm establishes a strong association between the system as a whole and the software”, "Until a certain point the ISO/IEC 12207 Norm is designed to act inside of a System Engineering process. When the software is part of a complete system, the software is isolated from the system, produced, and reintegrated into the system. When software constitutes all the scope of interest, the tasks in system level can be treated as a useful orientation.”.

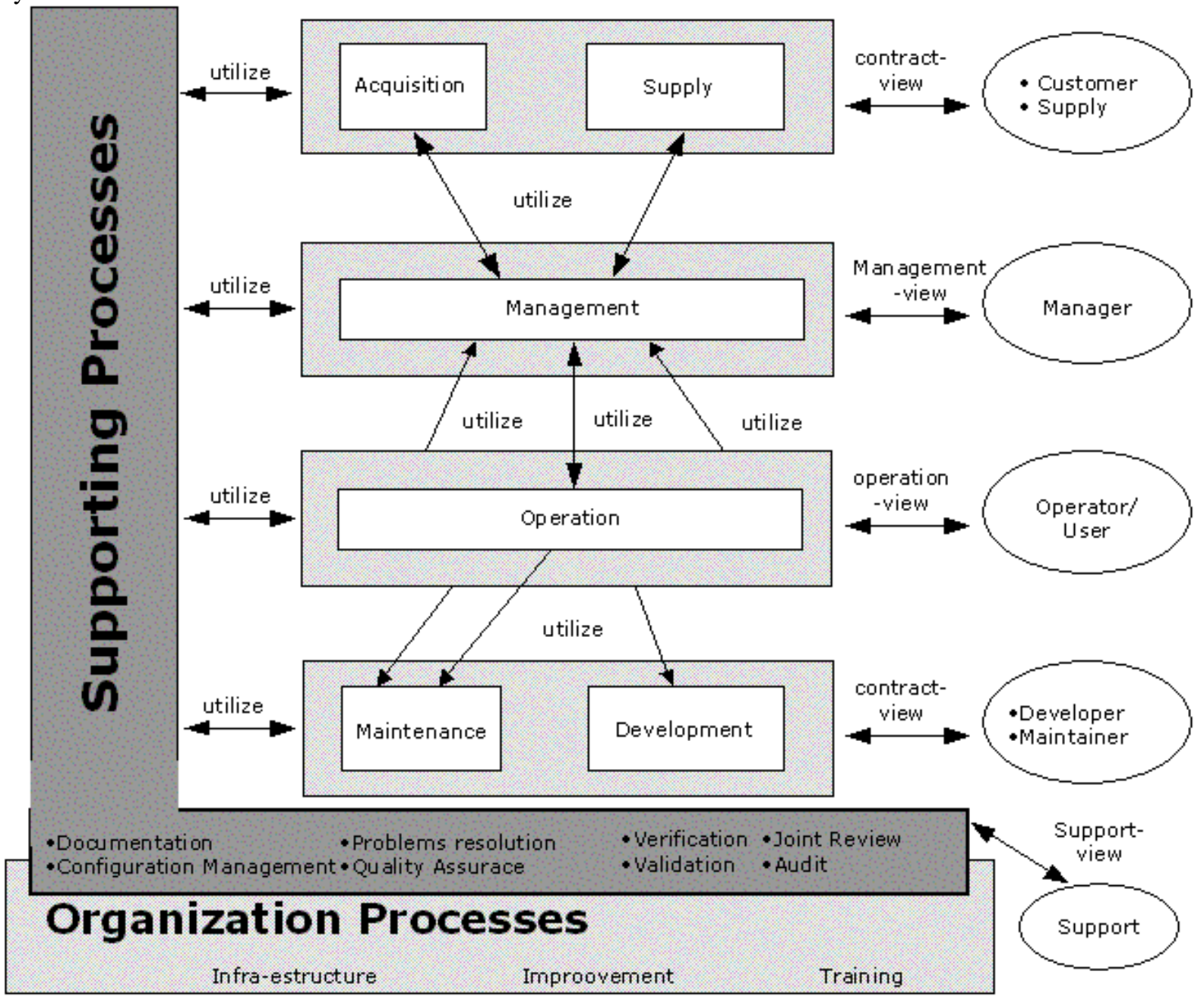

Figure 3: Software Lifecycle Processes - Rules and relationships 


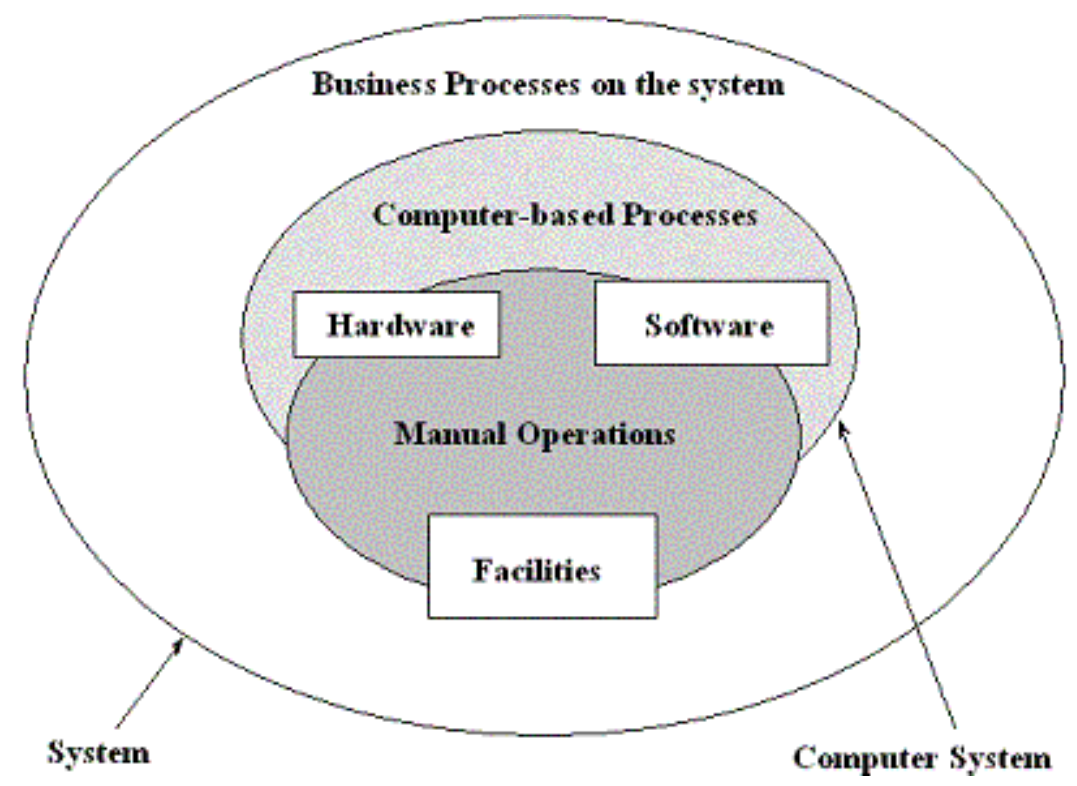

Figure 4. Software view in the system

\section{An ISO 9001:2000 CERTIFICATION MODEL}

The Quality Management System (QMS) with the focus on the software development actions concerning the quality processes and products, as also on satisfying to the necessities of the customers, must be discussed and adopted by the high level administration of the organization. What follows is a descriptions of the steps for a certification ISO 9001:2000:

a The implantation of the QMS should be realized using the 3 macro-processes of ISO/IEC 12207 Norm [4], serving as tripod for software development activities. This structure attends to the requirements of ISO 9001 Norm and reflects a systemic vision of the company and its routines, both the production routines and the administration routines. Thus, the described activities and day-to-day tasks of the software organization will assist their collaborators to identify and place their responsibilities in various formalized processes. The mapping of ISO 9001 with ISO/IEC 12207 primarily supports this identification, with ISO 9001 also incorporating the technical role of a software company. Using this premise, all the requirements for ISO 9001 certification were enclosed in the software life cycle processes, in addition to other important and necessary procedures that had been generated.

a Quality Planning should be realized using the directives determined by the organization's directors in the Quality Policies and should be coherent with the Quality Objectives. During this planning, the requirements of the quality management and the implementation of the necessary continuous improvements must be taken into consideration to attend to the Quality Policies and to maintain the integrity of the QMS. These improvements will be made operational by means of the implementation changes to the QMS, in a form to maintain it permanently up-to-date and in accord with the requirements specified for the customers.

a The activities that influence the quality directly or indirectly will be realized in structuralized form. This structure includes activities to verify and control the quality in the diverse stages of the processes, which will be realized in a planned manner and obey the requirements and instructions defined in the registered procedures of the QMS.

- The QMS should be maintained up-to-date and its effectiveness will be improved in a continuous manner, in accordance with the established Quality Policies and by the basic directives of the senior administration. Its characteristics must be described in documented procedures, which will be elaborated in a way that describes the methodology used to realize the related activities with the quality processes and products. The QMS document structure is composed of:

- Laws, decrees, edicts

- Quality Manual

- $\quad$ Procedures
(Legal documents)

(Main document of the QMS)

(Normative documents) 
- Tutorials

- Forms/Models

- $\quad$ Records
(Operational Documents)

(Support for registering data)

(Results/evidences)

a The Quality Manual must include a description of the purpose of the certification, the quality policies, references to the registered procedures used in the different activities related to the administrative and productive procedures and a description of the interaction between the QMS processes.

a The activities that influence directly or indirectly the quality of the processes or products must be described in the Tutorial Procedures and they will be used to assure the correct realization of activities and the fulfillment of the specified requirements. In the QMS of the software company, at least 13 registered procedures must exist:

- Organizational procedures: These contribute to the process of improvement of the company and are beyond of the domain of a single project.

$>$ Security Procedures;

$>$ Director Responsibilities Procedures;

$>$ Supplier Qualification Procedures;

$>$ Professional Qualification Procedures;

$>$ Strategic Qualification Program.

- Support Procedures: These assist and contribute to the success and the quality of the projects/products.

$>$ Document Control Procedure;

$>$ Record Control Procedure;

$>$ Internal Audits Procedures;

$>$ Non-conform Product Control Procedure;

> Corrective, Preventive and Improvement Actions Procedures;

$>$ QMS Description Procedures.

- Fundamental Procedures: Used directly in the software lifecycle.

> Systems Design and Development Procedures;

$>$ Software Development Methodology adopted by the Organization.

The procedures determine (or they make reference to), when necessary, the use of operational documents, technical documents and/or tutorials and forms to support data records. The necessary Quality Records must be defined in the different operational phases, as well as the applicable forms that support this information and data.

The responsibility for the management of the processes described in the registered procedures is defined in the proper body of each document, except in the case of forms where it is not required. When necessary, the implementation of the documents is preceded by training of the functions involved, ensuring the correct interpretation and application of the specified requirements.

\subsection{Script of Activities}

The effort to introduce a QMS and the posterior certification of the organization are divided into activities developed for the participants, and are related in Table 2. The activities of this table are ordered to represent the sequence in the process of certification ISO 9001:2000 of a SME.

These activities, for better understanding, are characterized in the following way:

- Local: Corresponding to the section of ISO 9001 where the activity is located. An activity unforeseen in the ISO, but considered necessary, is marked with " $\mathrm{N}$ ".

口 Mode: Sequential activity (S) or an activity developed in a parallel form (P).

- Type: Activity executed once (U), activity executed for the duration of the process of certification in a continuous form (C), activity execution is divided (D), and an activity that is repeated several times (R). 
Table 2: QMS-Project Activities

\begin{tabular}{|c|c|c|c|}
\hline ACTIVITIES & Local & Mode & Type \\
\hline Opening of works, presentation of the QMS project. & $N$ & $S$ & $U$ \\
\hline Analysis of the organization processes and products. & 4 & $P$ & $D$ \\
\hline Study of the organization processes of development. & 4 & $P$ & $D$ \\
\hline Study of the software products offered by the organization. & 4 & $P$ & $D$ \\
\hline Study of ISO 9000:2000 Norm. & $N$ & $P$ & $D$ \\
\hline Definition of the quality policies: Mission, Future Vision, Values and Principles. & 4 & $S$ & $U$ \\
\hline $\begin{array}{l}\text { Quality objectives: Define objectives, Establish goals, Establish terms, Establish responsibilities, } \\
\text { Establish standards and indicatives of performance. }\end{array}$ & 4 & $S$ & $U$ \\
\hline $\begin{array}{l}\text { To elaborate obligatory documented procedures required by the norm: Document control, Record } \\
\text { Control, non-conforming product control, corrective actions control, preventive actions control, } \\
\text { internal-audit control . }\end{array}$ & 4 & $S$ & $U$ \\
\hline $\begin{array}{l}\text { To elaborate non-obligatory documented procedures responsible for the software development } \\
\text { process. }\end{array}$ & $N$ & $S$ & $U$ \\
\hline To identify organizational processes in the Norm optics. & $N$ & $P$ & $C$ \\
\hline To identify the necessary records and to implement the required controls. & 4 & $S$ & $D$ \\
\hline To define and to allocate the necessary resources. & 6 & $S$ & $U$ \\
\hline $\begin{array}{l}\text { To identify the necessary requirements of abilities for the functions that affects the quality of the } \\
\text { processes. }\end{array}$ & 6 & $S$ & $U$ \\
\hline To verify necessary competencies of the human resources. & 6 & $S$ & $U$ \\
\hline To identify the necessary training. & 6 & $S$ & $U$ \\
\hline To verify the effectiveness of the training. & 6 & $S$ & $U$ \\
\hline To verify the necessary financial resources, infrastructure and environment. & 6 & $S$ & $U$ \\
\hline Implementation of the processes related to the product and software development. & 7 & $P$ & C \\
\hline Critical analysis by the high level administration. & 7 e 5 & $S$ & $D$ \\
\hline Validation and involvement of the high level administration. & 5 & $S$ & $D$ \\
\hline Internal Quality-Audit & 8 & $S$ & $R$ \\
\hline Improvement Processes. & 8 & $P$ & $C$ \\
\hline Corrective / Preventive Actions. & 8 & $P$ & $R$ \\
\hline Final adjustments. & 8 & $P$ & $U$ \\
\hline
\end{tabular}

The following activities must also be developed in the organization for the conclusion of the process of ISO 9001 certification:

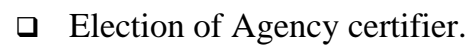

口 Pre-audit (Presence of the Auditor on organization)

口 Audit (Presence of the Auditor on organization)

Apart from the documented procedures previously cited, one of the products generated and validated fruits of the certification process was the elaboration of a Software Development Methodology for the software company.

\section{CONCLUSION}

This work presented the model used in the certification process of the ISO 9001:2000 Norm [2] of a Brazilian software company, SoftExport, a SME with approximately 21 collaborators including the 3 directors who assume development roles. The implemented QMS from the ISO/IEC 12207 Norm [4] has assured that its product development process has a disciplined, repeatable level of control, guaranteeing the quality of its products.

The certification process was conducted through a set of structured activities from ISO/IEC 12207 Norm, differentiating itself from the logical implementation sequence of the items in the ISO 9001 Norm, bringing a higher level of agility to the implementation process of the QMS within the software company. 
We can cite as the main lessons learned during the certification process ISO 9001:2000 [2]:

a The explicit support of the high level administration of the organization was of fundamental relevance, once it was opened to the participants of the certification process, all the structures of the company and of its collaborators, in a prioritized form.

a The definitions of Vision, Policy and Quality Objectives motivated the directors to define and to follow indicators, that was of real interest and motivated the control of the organizational structure;

- The analysis and study of the software development processes adopted by the organization has provided to the participants of the process, a fast integration with the routines and the developers;

a The analysis and the study of the organization's software products identified that several of them were used internally in the processes of management and development. Some of these products are already appropriate obligatory control items of the norm;

- The description of all the roles of the organizational structure, identifying their necessary responsibilities, activities and qualifications. Remembering that a collaborator can assume several of these roles;

- The study of ISO 9000:2000 [1] Norm positioned the group in the QMS environment. The approach developed was the identification and the adaptation of the routines, processes, methodologies and products of the company to the Norm. (Figure 5)

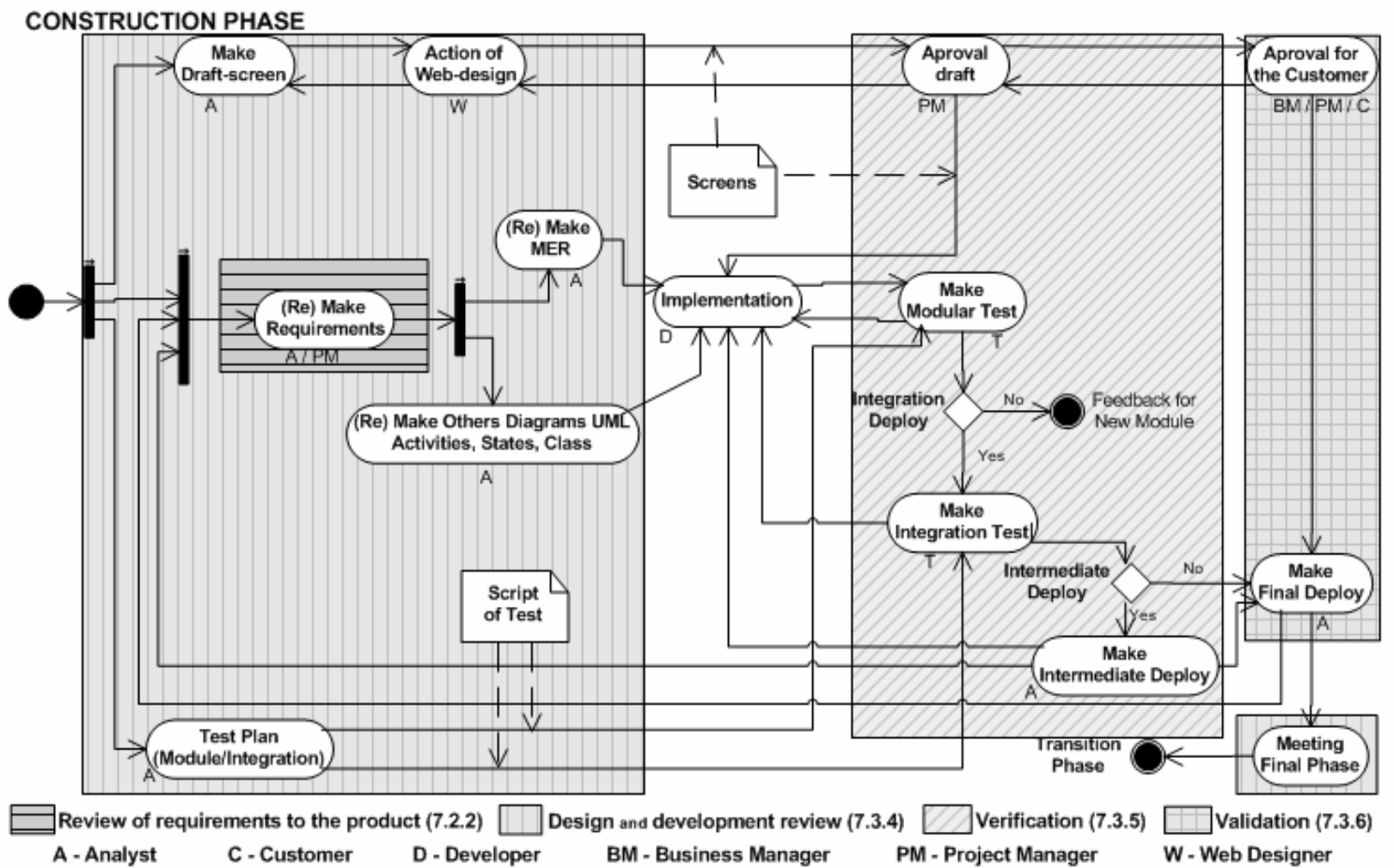

Figure 5: Construction Phase of internal methodology indicating the requisites of the ISO 9001 Norm

口 The mapping between ISO 9001:2000 [2] and ISO/IEC 12207 [4] integrated the certification requirements with the lifecycle processes, enabling the division into three action fronts (organizational, fundamental and support processes) and facilitating to the dialogue between all the organizational collaborators;

a The sequence of activities used in the ISO 9001:2000 certification process [2] for the software company, that was considered satisfactory, is presented as follows:

- $\quad$ QMS (section 4 of the Norm): the establishment, documentation and the implementation of a quality system describing the necessity of the processes to be known and documented.

- $\quad$ Resources Management (section 6): presents the available and necessary resources to practice quality.

- Accomplishment of the Product (section 7): shows to the implementation of the product with the focus on customer procedures and requirements. 
- Responsibility of the Direction (section 5): integrates the High Level Administration in the actual implementation process of the QMS, validating and adopting the quality process before all the collaborators.

- $\quad$ Measurement, Analysis and Improvement (section 8): describes the process of measurement, analysis and improvement, being able to return to any of the stages of implementation.

In this way, the project of implementation of the QMS based on ISO/IEC 12207 [4] can be seen as a process that follows ISO 9001 [2] and uses its activities of measurement, analysis and improvement (section 8), to adjust the other activities. The Direction Responsibility activity assures the validity and the reach of the proposals and reaffirms the necessary support all the process.

\section{References}

[1] ISO 9000. 2000a. Quality management systems - Fundamentals and vocabulary.

[2] ISO 9001. 2000b. Quality management systems - Requirements.

[3] Paduan, Roberta et al. 2003. Tesouro Escondido. EXAME. São Paulo. v.37, n.13.

[4] ISO/IEC 12207. 1995. Information Technology: Software life cycle processes. ISO, Geneva-Swiss.

[5] Rocha, A. R. C., Maldonado, J. C. e Weber, K. C. 2001. Qualidade de Software: Teoria e Prática, São Paulo. Prentice Hall.

[6] Weber. K. C., Rocha,A. R. C. e De Luca, J. C. M. 1999. Qualidade e Produtividade em Software . 2a. Edição. São Paulo. Makron Books.

[7] ISO/IEC 15271. 1998. Information Technology: Guide for ISO/IEC NBR 12207(Software life cycle processes). ISO. Geneva-Swisss.

[8] Li, Chao et al. 2001. A Software Factory Model on ISO 9000 and CMM for Chinese Small Organizations, IEEE.

[9] Campos, V. F. 1992. Qualidade de total: Padronização de empresas .Belo Horizonte, QFCO.

[10] ISO/IEC 12207.1995/Amd 1:2002. Information Technology: Software life cycle processes. ISO, Geneva-Swiss. 